\title{
Monitoramento avançado de enfermagem: pacientes de risco na atenção primária
}

\author{
Advanced nursing monitoring: At-risk patients in Primary Care \\ Monitoreo avanzado de enfermería: pacientes de riesgo en atención primaria
}

\begin{abstract}
Layse Farias $\mathrm{Nava}^{1}$ (1)
Tayse Tâmara da Paixão Duarte ${ }^{1}$ (1)

Wellington Luiz de Lima ${ }^{1}$ (D)

Marcia Cristina da Silva Magro $^{1}$ (D)
\end{abstract}

1. Universidade de Brasília, Programa de Pós-Graduação em Enfermagem. Brasília, DF, Brasil.

Autor correspondente:

Layse Farias Nava.

E-mail: layse.nava@live.com

Recebido em 09/07/2021.

Aprovado em 25/10/2021.

DOI:https://doi.org/10.1590/2177-9465-EAN-2021-0282

\section{Resumo}

Objetivo: avaliar o efeito do monitoramento de enfermagem avançado em relação ao usual para identificação do risco cardiovascular e renal, adesão medicamentosa e prática de exercícios na atenção primária à saúde. Método: estudo quantitativo, quase-experimental desenvolvido na Atenção primária. Em relação a intervenção, foram consultas de enfermagem trimestrais de monitoramento avançado e laboratorial trimestral. Para coleta de dados, aplicou-se questionário sociodemográfico, escala Morisky e Questionário Internacional de Atividade Física. Resultados: sexo feminino predominou nos dois grupos intervenção e controle $(62,79 \%$ vs. $76,74 \%)$. O percentual de pacientes sem risco cardiovascular do grupo intervenção superou o grupo controle da consulta 1 para consulta 3, (0,00\% - 25,58\% vs. 6,98 - 2,33). Adesão medicamentosa máxima, ao longo do tempo foi superior no grupo intervenção comparado ao grupo controle (48,8\% vs. $23,3 \%)$. O risco cardiovascular dos usuários interferiu na atividade física de forma significativa no grupo intervenção e controle ( $p=0,0261$ vs. 0,0438$)$. Conclusões e implicações para a prática: a monitorização avançada possibilitou uma melhor identificação de pacientes de risco e orientações aos pacientes hipertensos e diabéticos com risco cardiovascular e renal, o que favoreceu o monitoramento avançado e contribuiu ao autogerenciamento da prática de exercícios e adesão medicamentosa, a partir de consultas de enfermagem.

Palavras-chave: Atenção primária à saúde; Fatores de risco de doenças cardíacas; Prática avançada de enfermagem; Adesão à medicação; Monitoramento.

\section{Abstract}

Objective: to evaluate the effect of advanced versus usual Nursing monitoring to identify cardiovascular and renal risk, adherence to medication and practice of physical exercise in Primary Health Care. Method: a quantitative and quasi-experimental study developed in Primary Care. In relation to the intervention, there were quarterly Nursing advanced monitoring consultations and quarterly laboratory control. For data collection, a sociodemographic questionnaire, the Morisky scale and the International Physical Activity Questionnaire were applied. Results: the female gender predominated both in the intervention and in the control group $(62.79 \%$ vs. $76.74 \%)$. The percentage of patients without cardiovascular risk in the intervention group exceeded the control group from consultation 1 to consultation $3(0.00 \%-25.58 \%$ vs. 6.98 - 2.33). Maximum adherence to medication, over time, was higher in the intervention group when compared to the control group ( $48.8 \%$ vs. $23.3 \%)$. The users' cardiovascular risk significantly interfered with physical activity in the intervention and control groups ( $p=0.0261$ vs. 0.0438 ). Conclusions and implications for the practice: advanced monitoring enabled better identification of at-risk patients and guidelines for the hypertensive and diabetic patients at cardiovascular and renal risk, which favored advanced monitoring and contributed to self-management of the practice of physical exercise and adherence to medication, based on Nursing consultations.

Keywords: Primary health care; Risk factors for heart diseases; Advanced practice nursing; Adherence to medication; Monitoring

\section{Resumen}

Objetivo: evaluar el efecto de la monitorización avanzada de enfermería frente a la habitual para identificar el riesgo cardiovascular y renal, la adherencia a la medicación y la práctica del ejercicio en la atención primaria de salud. Método: estudio cuasiexperimental cuantitativo desarrollado en Atención Primaria. En cuanto a la intervención, se realizaron consultas de enfermería trimestrales para seguimiento avanzado y laboratorio trimestral. Para la recopilación de datos se aplicó un cuestionario sociodemográfico escala de Morisky y Cuestionario Internacional de Actividad Física. Resultados: el género femenino predominó tanto en el grupo de intervención como en el de control $(62,79 \%$ vs $76,74 \%)$. El porcentaje de pacientes sin riesgo cardiovascular en el grupo de intervención superó al grupo de control desde la cita 1 hasta la cita $3(0,00 \%-25,58 \%$ frente a 6,98 - 2,33). La adherencia máxima al fármaco, a lo largo del tiempo, fue mayor en el grupo de intervención en comparación con el grupo de control ( $48,8 \%$ frente a $23,3 \%$ ). El riesgo cardiovascular de los usuarios interfirió significativamente con la actividad física en los grupos de intervención y control ( $p=0,0261$ frente a 0,0438 ). Conclusiones e implicaciones para la práctica: la monitorización avanzada permitió identificar mejor a los pacientes en riesgo y orientar a los pacientes hipertensos y diabéticos con riesgo cardiovascular y renal, lo que favoreció el monitoreo avanzado y contribuyó al autocontrol de la práctica de ejercicio y la adherencia a la medicación desde las consultas de enfermería.

Palabras clave: Atención primaria de salud; Factores de riesgo de enfermedad cardiaca; Enfermería de práctica avanzada; Adherencia a la medicación; Monitoreo. 


\section{INTRODUÇÃO}

O monitoramento de usuários do sistema de saúde pode ser uma estratégia que contribui para mudanças oportunas e que facilita o estabelecimento de metas de colaboração direta com os pacientes ao se desenvolver planos de cuidado centrado nas necessidades do paciente ${ }^{1}$. Portanto, acredita-se que essa estratégia seja promissora, para aumentar a capacidade de autogerenciamento de doenças crônicas não transmissíveis, como o diabetes mellitus e a hipertensão arterial, sendo de suma importância na melhoria da qualidade de vida relacionada à saúde, na adesão a práticas de exercício e na adesão medicamentosa².

Os elevados gastos relacionados às estratégias de controle do diabetes mellitus e hipertensão arterial, atualmente, impõem um fardo substancial à economia global e como resultado o aumento dos custos diretos e indiretos ${ }^{3,4}$, que inclusive podem ser impactados pelo aumento da expectativa de vida, e consequente aumento do número de pessoas com múltiplas doenças crônicas (multimorbidade). Pacientes com multimorbidade requerem cuidados de saúde especializados, modelo promissor oferecido na atenção primária como um diferencial para gerenciamento e monitoramento das doenças ${ }^{5}$ e consequente menor tempo de espera e prevenção da hospitalização.

Nesse contexto, modelos de atendimento, como a prática avançada de enfermagem na atenção primária, constituída por enfermeiros com conhecimento especializado e habilidades para tomada de decisões e competências clínicas, podem ser determinantes para melhor gerenciamento das necessidades dos pacientes, além de cuidados de alta qualidade ${ }^{6}$.

Entretanto, ainda parece haver um distanciamento entre o cuidado esperado e o prestado pelas equipes de saúde. Intervenções profissionais parecem ser alternativas para redução de lacunas entre esses modelos de cuidado ${ }^{7,8}$. Sabe-se que o modelo biomédico concentra esforços no bem-estar físico, sendo assim adotar modelos com abordagem holística parece mostrar maior alinhamento a premissa da Organização mundial de Saúde (OMS), a qual contempla a saúde não apenas como ausência de doença ou enfermidade ${ }^{9}$. Enquanto fenômeno ampliado, considera-se que a saúde envolve modos de ser e produzir e/ou recriar a vida em sua singularidade e multidimensionalidade ${ }^{10}$.

Revisão sistemática aponta que profissionais de saúde, inclusive os enfermeiros na atenção primária, estão qualificados para apoiar a prestação de autocuidado do paciente de forma estruturada e baseada em evidências. A melhor compreensão dos resultados de um monitoramento eficaz pode ser beneficiada pela integração desses profissionais, o que pode gerar mudanças no sistema de atenção primária à saúde ${ }^{11}$, melhorar a satisfação dos pacientes, complicações e atenuar os fatores de risc $0^{5}$ relacionados a doença cardiovascular e renal, geralmente, presentes em pacientes com diabetes mellitus e hipertensão arterial.

Nesse sentido, o objetivo deste estudo foi avaliar o efeito do monitoramento de enfermagem avançado em relação ao usual para identificação do risco cardiovascular e renal, adesão medicamentosa e prática de exercícios na atenção primária à saúde.

\section{MÉTODO}

Estudo quantitativo, quase-experimental, não equivalente desenvolvido em uma unidade básica de saúde da região Oeste de Brasília, Distrito Federal, Brasil.

A amostra foi por conveniência e constituiu-se de 86 usuários cadastrados no programa saúde da família, sendo 43 do grupo intervenção e 43 do grupo controle com diagnóstico médico de diabetes mellitus e hipertensão arterial sistêmica. O acompanhamento ocorreu no período de fevereiro a dezembro de 2019.

O tamanho amostral obtido por meio do software IBM SPSS Sample Power versão 3.0 foi de 32 usuários/grupo, adotandose um $a=5 \%$ e o $\beta=80 \%$ e os percentuais de disfunção renal encontrados no teste piloto, realizado com dez usuários de saúde. Considerando $20 \%$ de perdas, o tamanho amostral foi de pelo menos 40 usuários em cada grupo.

O estudo foi desenvolvido em uma das Unidades Básicas de Saúde (UBS) da região Oeste de Brasília, Distrito Federal, Brasil. A equipe de profissionais atuante nessa UBS constituía-se à época de cinco agentes comunitários de saúde, dois técnicos de enfermagem, um enfermeiro, um dentista e um médico. Os serviços complementares e integrados ao modelo de cuidado disponibilizado à população adscrita nessa unidade eram Yoga Hatha, terapia comunitária integrativa, saúde e bem-estar, saúde da coluna (fisioterapia), saúde do assoalho pélvico (fisioterapia) e saúde do idoso (fisioterapia).

Os critérios de inclusão foram usuários com diagnóstico médico de hipertensão arterial e diabetes mellitus tipo 2 e capacidade cognitiva preservada avaliada pelo nível de consciência à época da seleção para o estudo. Foram excluídos usuários com incapacidade física para deslocamento e que não compareceram às consultas de enfermagem para monitoramento das condições clínicas cardiovasculares, renais, adesão medicamentosa e a prática de exercícios.

Em relação a intervenção, foram consultas de enfermagem trimestrais realizadas durante o período de nove meses com monitoramento avançado laboratorial trimestral, das condições clínicas, da adesão medicamentosa e da prática de exercícios e no grupo controle o mesmo monitoramento foi mantido, mas sem controle laboratorial trimestral (modelo usual). Para cada paciente realizou-se uma consulta com intervalo de três meses, o que somou um total de três consultas de enfermagem.

As variáveis de interesse foram, idade, sexo, escolaridade (ensino fundamental, médio e superior), grau de instrução, estado civil, raça, com quem reside (família, filhos, irmãos, outros), comorbidades, parâmetros hemodinâmicos (pressão arterial sistólica e diastólica), glicemia capilar, hemoglobina glicada e creatinina séricas, clearance de creatinina, hábitos de vida, ocupação, prática de atividade física, adesão medicamentosa. Foram utilizados para coleta de dados um questionário sociodemográfico estruturado elaborado pelos pesquisadores, escala Morisky ${ }^{12}$ e Questionário Internacional de Atividade Física (IPAQ) $)^{13}$ validados no Brasil.

A investigação da adesão medicamentosa foi realizada ao final de cada consulta por meio do Teste de Morisky e Green 
de consistência interna de $0,61^{12}$ que possibilita determinar a adesão ou não adesão. É constituído de quatro perguntas às quais os entrevistados respondem de forma dicotômica, isto é, "sim/não" para as seguintes indagações: 1) $\mathrm{O}(\mathrm{a}) \mathrm{Sr}(\mathrm{a})$ alguma vez esqueceu de tomar seus medicamentos?; 2) $\mathrm{O}$ (a) $\mathrm{Sr}$ (a) foi descuidado com os horários de tomar seus medicamentos?; 3) Quando o(a) Sr(a) sentiu-se melhor, às vezes, parou de tomar seus medicamentos?; 4) Alguma vez, quando o(a) $\mathrm{Sr}$ (a) se sentiu mal, parou de tomar seus medicamentos?

Assim, considera-se no Teste Morisky que um SIM equivale a zero ponto, enquanto um NÃO equivale a um ponto. Desse modo, possui alto grau de adesão (máxima adesão) aquele usuário cujas respostas forem todas negativas, totalizando quatro pontos. Para moderado grau de adesão, quando o total de respostas totalizar três pontos e baixo grau de adesão quando forem totalizados um ou dois pontos. Já a classificação "não aderente" ocorre quando todas as respostas são positivas, totalizando zero ponto ${ }^{12}$.

O IPAQ é um questionário que permite estimar o tempo semanal gasto em atividades físicas de intensidade moderada e vigorosa, em diferentes contextos do cotidiano, como: trabalho, transporte, tarefas domésticas e lazer, e ainda o tempo despendido em atividades passivas, realizadas na posição sentada. Utilizouse a versão reduzida composta por sete questões abertas com informações que permitem estimar o tempo despendido, por semana, em diferentes dimensões de atividade física (caminhadas e esforços físicos de intensidades moderada e vigorosa) e de inatividade física (posição sentada) ${ }^{13,14}$. O nível de atividade física foi avaliado uma vez ao final de cada consulta de enfermagem a partir da pontuação obtida pela soma da quantidade de dias e minutos ou horas das atividades físicas realizadas na semana anterior ao preenchimento do questionário, sendo assim foram classificados em: muito ativo (Atividade VIGOROSA: $\geq 5$ dias na semana e $\geq 30$ minutos por sessão; ou Atividade VIGOROSA: $\geq 3$ dias na semana e $\geq 20$ minutos por sessão + Atividade MODERADA e/ou CAMINHADA: $\geq 5$ dias na semana $e \geq 30$ minutos por sessão. Ativo (Atividade VIGOROSA: $\geq 3$ dias na semana e $\geq 20$ minutos por sessão ou Atividade MODERADA ou CAMINHADA: $\geq 5$ dias na semana e $\geq 30$ minutos por sessão ou qualquer atividade somada: $\geq 5$ dias na semana $e \geq 150$ minutos na semana (caminhada + atividade moderada + atividade vigorosa). Irregularmente ativo A (frequência: 5 dias na semana; ou Duração: 150 minutos na semana). Irregularmente ativo $B$ (aquele que não atingiu nenhum dos critérios da recomendação quanto à frequência nem quanto à duração). Sedentário (aquele que não realizou nenhuma atividade física por pelo menos 10 minutos contínuos durante a semana) ${ }^{13}$.

\section{PROCEDIMENTOS DE COLETA DE DADOS}

Os usuários cadastrados como hipertensos e diabéticos foram convidados a participar da pesquisa com mediação dos agentes comunitários.

Agendou-se uma reunião na unidade básica de saúde para apresentação da pesquisa e seus objetivos. A impossibilidade de garantir a aleatoridade no processo de alocação dos usuários em grupo intervenção ou controle é uma característica do quase experimento e esteve relacionada ao processo de seleção baseado no número de cadastro do usuário na rede de atenção primária.

Os usuários que concordaram em participar do estudo assinaram o Termo de Consentimento Livre e Esclarecido. A classificação dos usuários nos grupos controle e experimental obedeceu ao critério do número da matrícula da secretaria de saúde do Distrito Federal, e a sua combinação numérica final, quando par, indicava o usuário ao grupo experimental e quando ímpar o usuário foi direcionado ao grupo controle. Ao término, cada usuário foi orientado quanto a data de agendamento da primeira consulta de enfermagem.

$\mathrm{Na}$ consulta, o pesquisador realizava mensuração da pressão arterial e da glicemia capilar e aplicava o questionário e as escalas Morisky ${ }^{12}$ e IPAQ ${ }^{13}$ para avaliação da adesão medicamentosa e da prática de atividade física, respectivamente. Também foi realizado medidas antropométricas, como peso e altura e investigação clínica com exame físico. O tempo de duração da consulta foi em média de 50 minutos. Nesse período, orientações sobre medidas de autocuidado, como controle da pressão arterial, do peso e da glicemia eram fornecidas para melhor controle dos riscos advindos da hipertensão arterial e diabetes, importância da prática de exercícios físicos, controle alimentar e sensibilização para adesão medicamentosa.

Durante a consulta de enfermagem no consultório a mensuração da pressão arterial sistêmica foi realizada com esfigmomanômetro manual da marca P.A.MED® e o usuário na posição sentada, após repouso de 5 minutos ${ }^{15}$. A mensuração foi repetida para confirmação quando se identificou alterações dos valores da pressão sistólica < 90 ou > $140 \mathrm{mmHg}$ e da pressão diastólica $<60$ ou $>100 \mathrm{mmHg}^{16}$.

A glicemia capilar pós-prandial foi avaliada pelo aparelho da marca Accu-Chek $₫$ do modelo Active e lancetas da marca G-Tech®, após antissepsia da polpa de um dos dedos da mão e punção com a lanceta. Os parâmetros de normalidade da glicemia de jejum foram $<100 \mathrm{mg} / \mathrm{dL}$ e pós-prandial $<160 \mathrm{mg} / \mathrm{dL}^{16}$.

Para medida do peso corporal, primeiramente, solicitouse a retirada do excesso de roupas, sapatos e acessórios e o esvaziamento da bexiga e quanto a estatura o usuário foi orientado a posicionar-se na balança digital Balmak BK300FAN®. A estatura foi medida após posicionamento da região dorsal do usuário na régua da balança sem calçados ${ }^{15}$.

A circunferência abdominal (CA) foi medida para avaliação do risco cardiovascular no final da expiração por meio de fita métrica graduada em centímetros com o usuário em posição supina e identificação do ponto médio entre a última costela e a crista ilíaca. Significou risco cardiovascular aumentado para mulheres a circunferência abdominal $>80 \mathrm{~cm}$ e para os homens a circunferência abdominal $>94 \mathrm{~cm}^{17}$.

Os parâmetros laboratoriais de referência foram: creatinina sérica $0,70-1,2 \mathrm{mg} / \mathrm{dL}$, ureia sérica $10,0-50,0 \mathrm{mg} / \mathrm{dl}$, clearence de creatinina para mulheres: 88 a $128 \mathrm{~mL} / \mathrm{min} / 1,73 \mathrm{~m}^{2}$ e homens: 97 a $137 \mathrm{~mL} / \mathrm{min} / 1,73 \mathrm{~m}^{2}$ e a hemoglobina glicada (HbA1c): 
baixo risco para diabetes, valor menor $5,7 \%$, risco aumentado para diabetes, valor de 5,7 a 6,4\% e diabéticos, valor maior que $6,4 \%$, conforme protocolo da Secretaria de Saúde do Distrito Federal-Brasil ${ }^{16}$.

Para os usuários do grupo intervenção diferente do grupo controle ao final da consulta fornecia-se um voucher no valor da passagem de transporte público de ida e volta para coleta de exames laboratoriais (glicemia de jejum, hemoglobina glicada, creatinina séricos e clearance de creatinina), a partir de fomento público. Para os usuários do grupo controle, a dosagem dos exames laboratoriais era realizada na rotina usual (uma vez no semestre) da própria unidade básica.

O risco cardiovascular foi avaliado pela calculadora de Risco de Framingham, que inclui os seguintes itens: (1) idade, (2) sexo, (3) história de tabagismo, (4) presença de diabetes, (5) pressão arterial sistólica (PAS), (6) se um paciente está atualmente em uso de medicação para reduzir a pressão arterial, (7) colesterol total e (8) colesterol HDL. Esta é uma ferramenta de estratificação de risco validada que estabelece o risco de um paciente desenvolver doença cardiovascular ${ }^{18}$.

A lesão renal aguda (LRA) foi avaliada a partir da classificação Kidney Disease: Improving Global Outcomes (KDIGO), definida pelo aumento de $\mathrm{s} \mathrm{Cr} \geq 1,5$ vezes da linha de base, ocorrido nos últimos 7 dias $^{19}$. A creatinina sérica baseline foi o menor valor no período de até 365 dias $^{20}$ pré-consulta de enfermagem. 0 volume urinário, ainda que conste como critério do KDIGO, não foi monitorado pela dificuldade de mensuração precisa no cenário da APS. Essa classificação permitiu a identificação e estadiamento da LRA: (1) estágio 1 (gravidade leve) quando há aumento de 1,5 a 1,9 vezes ou maior que $0,3 \mathrm{mg} / \mathrm{dL}$ do valor da creatinina basal; (2) estágio 2 (gravidade intermediária), quando há aumento de 2,0 a 2,9 vezes na creatinina basal; e (3) o estágio 3 (gravidade elevada) caracterizado pelo aumento de 3,0 vezes o valor basal da creatinina sérica ou aumento de $4,0 \mathrm{mg} / \mathrm{dL}^{19}$.

A identificação de alguma alteração clínica em qualquer uma das consultas de enfermagem gerava o encaminhamento do usuário ao profissional especializado para condutas direcionadas e específicas ao problema detectado para traçar um plano de cuidados individualizado, com a equipe de saúde que era responsável pelo paciente.

Foi realizada análise descritiva das variáveis de caracterização pelo cálculo da frequência absoluta e relativa e para descrever e comparar os itens dos constructos foi realizado cálculo das medidas de posição, tendência central e dispersão com cálculo de mediana, médias, erro padrão (EP) e percentis 25 e 75, para variáveis quantitativas contínuas. A homogeneidade entre grupos foi avaliada pelos testes Fisher e Qui-quadrado. Realizou-se o teste Kolmogorov-smirnov e identificou-se assimetria. Foi utilizado para comparações entre variáveis categóricas o teste Exato de Fisher ou sua extensão conhecida por Fisher-Freeman-Halton para tabela de contingência maior que $2 \times 2$. Para relacionar adesão medicamentosa nas diferentes consultas de enfermagem utilizou-se o teste de Friedman que permite detectar diferenças entre medianas de mais de duas amostras emparelhadas. $\mathrm{O}$ programa estatístico R versão 3.6.1 foi utilizado e considerou-se significativo a de 0,05.

Atendendo a Resolução 466/2012, este estudo foi aprovado pelo Comitê de Ética em Pesquisa da Fundação Ensino e Pesquisa em Ciências da Saúde (FEPECS), sob CAAE: 46509915.3.0000.5553, conforme Resolução 466/2012

\section{RESULTADOS}

Participaram do estudo 86 usuários, sendo 43 do grupo controle e 43 do grupo experimental, sendo predominante a ausência de diferença significativa entre os grupos pelo teste de homogeneidade. A idade mediana do grupo controle foi de 60 (41-66) anos e 59 (47,5-69) anos do grupo experimental. O sexo feminino foi predominante ( $76,74 \%$ vs. $62,79 \%)$ em ambos os grupos, respectivamente. $O$ ensino fundamental foi declarado pela maioria $(74,41 \%$ vs. $58,14 \%)$, assim como estado civil casado (58,14\% vs. $67,44 \%)$. O exercício de atividade profissional foi predominante no grupo intervenção $(44,19 \%)$, assim como história de etilismo e tabagismo ( $41,86 \%$ vs. $48,84 \%)$. A dislipidemia e a depressão foram mais, frequentemente, declaradas no grupo controle $(51,16 \%$ e $34,88 \%)$, respectivamente (Tabela 1$)$.

Ressalta-se que o nível sérico médio da glicose entre o grupo intervenção e controle foi similar $109 \mathrm{mg} / \mathrm{dL}(E P=6,71)$ vs. $105 \mathrm{mg} / \mathrm{dL}$ ( $E P=20,0)$, diferente da hemoglobina glicada 6,50 $(E P=0,3)$ vs. $8,10(E P=3,58)$ e da creatinina sérica média $(0,84$; $\mathrm{EP}=0,05 \mathrm{mg} / \mathrm{dL}$ vs. 0,$90 ; \mathrm{EP}=0,28 \mathrm{mg} / \mathrm{dL}$ ). A pressão arterial sistólica média foi superior no grupo controle em relação ao grupo intervenção $(135,31 ; \mathrm{EP}=3,25 \mathrm{mmHg}$ vs 126,55; $\mathrm{EP}=$ $3,11 \mathrm{mmHg})$ e a diastólica, ao contrário $(79,42 ; \mathrm{EP}=3,29 \mathrm{mmHg}$ vs. 82,43 ; $\mathrm{EP}=2,42 \mathrm{mmHg}$ ).

Observou-se que $9,52 \%$ dos pacientes do grupo controle evoluíram com risco para LRA e 4,76\% com lesão e falência renal, respectivamente durante o período de acompanhamento. O grupo intervenção não apresentou risco, tampouco evoluiu com LRA ao longo do acompanhamento.

Observa-se que dentre os níveis de risco cardiovascular, o grupo intervenção ao longo das consultas mostrou aumento da condição sem risco ( $0,00 \%$ vs. $25,58 \%)$. No grupo controle ocorreu o contrário, dos $6,98 \%$ sem risco na consulta 1 passou a $2,33 \%$ na terceira consulta. Vale destacar que não havia pacientes de alto risco cardiovascular no grupo intervenção e no controle o percentual foi baixo, sem modificação do padrão ao longo do acompanhamento $(2,33 \%)$. No grupo controle o número de usuários de risco baixo aumentou $(27,92 \%$ vs. $55,81 \%)$ e o risco médio diminuiu ( $62,79 \%$ vs. $39,53 \%)$. No grupo intervenção o risco baixo pouco reduziu ( $48,84 \%$ vs. $41,86 \%)$, assim como o risco médio $(51,16 \%$ vs. $32,56 \%)$ da primeira para a terceira consulta (Tabela 2).

Observou-se com o teste Morisky-Green que a maioria dos usuários dos grupos controle e experimental ainda relatava na terceira consulta, esquecimento para tomada de medicamentos ( $65,1 \%$ vs. $48,8 \%$ ), com maior expressividade para o grupo controle. $\mathrm{O}$ descuido para tomar os medicamentos (item 2) foi 
Tabela 1. Análise descritiva e de homogeneidade das variáveis de identificação do usuário. Brasília, 2019.

\begin{tabular}{|c|c|c|c|c|c|c|}
\hline & \multirow{2}{*}{ Variáveis } & \multicolumn{2}{|c|}{ Grupo intervenção } & \multicolumn{2}{|c|}{ Grupo controle } & \multirow{2}{*}{ Valor-p } \\
\hline & & $\mathrm{N}$ & $\%$ & $\mathrm{~N}$ & $\%$ & \\
\hline \multirow{2}{*}{ Escolaridade } & Ensino Fundamental & 25 & 58,14 & 32 & 74,41 & \multirow{2}{*}{0,361} \\
\hline & Ensino Superior & 7 & 16,27 & 5 & 11,63 & \\
\hline Sexo & Feminino & 27 & 62,79 & 33 & 76,74 & 0,240 \\
\hline Ativo na profissão & Sim & 19 & 44,19 & 13 & 30,23 & 0,285 \\
\hline \multirow{3}{*}{ Estado civil } & Casado & 29 & 67,44 & 25 & 58,14 & \multirow{3}{*}{0,806} \\
\hline & Solteiro & 8 & 18,60 & 11 & 25,58 & \\
\hline & Viúva & 4 & 9,30 & 4 & 9,30 & \\
\hline \multirow[t]{2}{*}{ Raça } & Pardo & 26 & 60,47 & 27 & 62,79 & \multirow[t]{2}{*}{0,836} \\
\hline & Preto & 8 & 18,60 & 6 & 13,95 & \\
\hline \multirow{4}{*}{ Faz diariamente } & Atividade Doméstica & 23 & 53,49 & 31 & 72,09 & \multirow{4}{*}{0,307} \\
\hline & Cuida dos netos & 2 & 4,65 & 1 & 2,33 & \\
\hline & Trabalha & 17 & 39,53 & 10 & 23,26 & \\
\hline & Free lance & 1 & 2,33 & 1 & 2,33 & \\
\hline \multirow{2}{*}{ Reside com quem } & Companheiro/Companheira & 8 & 18,60 & 4 & 9,30 & \multirow{2}{*}{0,341} \\
\hline & Família & 23 & 53,49 & 30 & 69,77 & \\
\hline Álcool & Sim & 8 & 18,60 & 4 & 9,30 & 0,002 \\
\hline \multicolumn{7}{|l|}{ Comorbidade } \\
\hline $\begin{array}{l}\text { Acidente vascular } \\
\text { encefálico }\end{array}$ & & 3 & 6,98 & 6 & 13,95 & 0,483 \\
\hline Doença renal & & 2 & 4,65 & 4 & 9,30 & 0,676 \\
\hline Dislipidemia & & 10 & 23,26 & 22 & 51,16 & 0,014 \\
\hline Depressão & & 10 & 23,26 & 15 & 34,88 & 0,342 \\
\hline
\end{tabular}

Teste de Fisher; Teste Qui-Quadrado

Fonte: Dados de Pesquisa.

maior no grupo controle em relação ao de intervenção $(46,5 \%$ vs. $34,9 \%$ ) (Tabela 3).

Nos dois grupos, o percentual referente a interrupção da tomada de medicamento por sentir-se mal reduziu ao longo das consultas, sendo no grupo controle $(14,0 \%$ para $9,3 \%$ ) e no intervenção (16,3\% para 11,6\%) (Tabela 3$)$.
A adesão medicamentosa máxima na terceira consulta foi expressa, principalmente, no grupo intervenção em relação ao grupo controle (48,8\%vs. 23,3\%). A ausência de adesão reduziu tanto no grupo controle como no grupo intervenção, de forma proporcional $(4,7 \%$ para $2,3 \%$ ) vs. ( $9,3 \%$ para $4,7 \%)$, ainda que sem diferença estatística (Tabela 4). 
Tabela 2. Classificação de risco cardiovascular nas diferentes consultas do grupo controle e intervenção. Brasília, 2019.

\begin{tabular}{|c|c|c|c|c|c|c|c|c|c|c|c|c|}
\hline \multirow{3}{*}{$\begin{array}{c}\text { Risco } \\
\text { cardiovascular }\end{array}$} & \multicolumn{6}{|c|}{ Grupo Intervenção } & \multicolumn{6}{|c|}{ Grupo Controle } \\
\hline & \multicolumn{2}{|c|}{ Consulta 1} & \multicolumn{2}{|c|}{ Consulta 2} & \multicolumn{2}{|c|}{ Consulta 3} & \multicolumn{2}{|c|}{ Consulta 1} & \multicolumn{2}{|c|}{ Consulta 2} & \multicolumn{2}{|c|}{ Consulta 3} \\
\hline & $\mathrm{N}$ & $\%$ & $\mathrm{~N}$ & $\%$ & $\mathrm{~N}$ & $\%$ & $\mathrm{~N}$ & $\%$ & $\mathrm{~N}$ & $\%$ & $\mathrm{~N}$ & $\%$ \\
\hline Alto & - & - & - & - & - & - & 1 & 2,33 & 1 & 2,33 & 1 & 2,33 \\
\hline Baixo & 21 & 48,84 & 21 & 48,84 & 18 & 41,86 & 12 & 27,91 & 24 & 55,81 & 24 & 55,81 \\
\hline Médio & 22 & 51,16 & 20 & 46,51 & 14 & 32,56 & 27 & 62,79 & 17 & 39,53 & 17 & 39,53 \\
\hline Sem risco & 0 & 0,00 & 2 & 4,65 & 11 & 25,58 & 3 & 6,98 & 1 & 2,33 & 1 & 2,33 \\
\hline
\end{tabular}

Fonte: Dados de Pesquisa.

Tabela 3. Distribuição das respostas relacionadas adesão à terapia medicamentosa (Morisky-Green) nos pacientes hipertensos e diabéticos. Brasília, DF, 2019.

\begin{tabular}{|c|c|c|c|c|c|c|c|c|c|}
\hline \multirow{2}{*}{$\begin{array}{c}\text { Grupos } \\
\text { Consulta }\end{array}$} & \multicolumn{3}{|c|}{ Controle } & \multicolumn{3}{|c|}{ Intervenção } & \multicolumn{3}{|c|}{ Total } \\
\hline & $\mathrm{C} 1$ & $\mathrm{C} 2$ & C3 & $\mathrm{C} 1$ & $\mathrm{C} 2$ & C3 & $\mathrm{C} 1$ & $\mathrm{C} 2$ & C3 \\
\hline Questões & $\operatorname{Sim} n(\%)$ & $\operatorname{Sim} n(\%)$ & Sim n(\%) & $\operatorname{Sim} n(\%)$ & $\operatorname{Sim} n(\%)$ & $\operatorname{Sim} n(\%)$ & $\operatorname{Sim} n(\%)$ & Sim $n(\%)$ & $\operatorname{Sim} n(\%)$ \\
\hline $\begin{array}{l}\text { O(a) Sr(a) alguma vez } \\
\text { esqueceu de tomar } \\
\text { seus medicamentos? }\end{array}$ & $19(44,2)$ & $18(41,9)$ & $28(65,1)$ & $25(58,1)$ & $20(46,5)$ & $21(48,8)$ & $44(51,2)$ & $38(44,2)$ & $49(57)$ \\
\hline $\begin{array}{l}\text { O(a) Sr(a) foi } \\
\text { descuidado com os } \\
\text { horários de tomar seus } \\
\text { medicamentos? }\end{array}$ & $16(37,2)$ & $14(32,6)$ & $20(46,5)$ & $16(37,2)$ & $16(37,2)$ & $15(34,9)$ & $32(37,2)$ & $30(34,9)$ & $35(40,7)$ \\
\hline $\begin{array}{l}\text { Q3 Quando o(a) Sr(a) } \\
\text { sentiu-se melhor, às } \\
\text { vezes, parou de tomar } \\
\text { seus medicamentos? }\end{array}$ & $8(18,6)$ & $6(14,0)$ & $6(14,0)$ & $9(20,9)$ & $10(23,3)$ & $10(23,3)$ & $17(19,8)$ & $16(18,6)$ & $16(18,6)$ \\
\hline $\begin{array}{l}\text { Alguma vez, quando } \\
\text { o(a) Sr(a) se sentiu mal, } \\
\text { parou de tomar seus } \\
\text { medicamentos? }\end{array}$ & $6(14,0)$ & $9(20,9)$ & $4(9,3)$ & $7(16,3)$ & $9(20,9)$ & $5(11,6)$ & $13(15,1)$ & $18(20,9)$ & $9(10,5)$ \\
\hline
\end{tabular}

C-Consulta.

Fonte: Dados de Pesquisa.

Os resultados mostraram que o risco cardiovascular interferiu significativamente no nível de atividade física tanto do grupo controle $(p=0,0438)$ como do grupo intervenção $(p=0,0261)$ (Tabela 5).

\section{DISCUSSÃO}

Os achados mostraram que o monitoramento avançado integrado às consultas de enfermagem foi mais efetivo para o controle do risco cardiovascular e renal, também da adesão medicamentosa e avaliação da prática de exercícios. Revisão sistemática mostrou que orientações fornecidas face a face favorecem o autogerenciamento clínico do paciente e melhor planejamento de ações ${ }^{11}$.

Nesse contexto, o (a) enfermeiro (a) no exercício de práticas de cuidado colaborativas desempenha ação fundamental no gerenciamento avançado de doenças crônicas, a partir do controle e monitoramento de fatores de risco, avaliação qualificada de tratamentos e medicamentos a partir de um trabalho informativo que influencia as políticas públicas ${ }^{21,22}$.

No entanto, a multimorbidade é compreendida como um desafio para o autogerenciamento do cuidado, especialmente, em condições como no estudo, agravadas pela elevada incidência de fatores de risco cardiovascular, dado que os pacientes do estudo além de hipertensos e diabéticos relataram como comorbidades o acidente vascular encefálico tanto no grupo controle $(13,95 \%)$ como intervenção (6,98\%), dislipidemia ( $23,26 \%$ vs. $51,16 \%$ ) e doença renal $(9,30 \%$ vs. $4,65 \%)$, respectivamente. No entanto, no campo da doença renal, destaca-se que somente no grupo controle se detectou ao longo do acompanhamento, pacientes com risco para lesão renal aguda $(9,52 \%)$, lesão e falência renal $(4,76 \%)$, respectivamente, conforme classificação KDIGO ${ }^{19}$. 
Tabela 4. Caracterização da adesão à terapia medicamentosa (Morisky-Green) nos pacientes hipertensos e diabéticos. Brasília, DF, 2019.

\begin{tabular}{|c|c|c|c|c|c|c|c|c|}
\hline Morisky & \multicolumn{3}{|c|}{ Controle } & \multirow{3}{*}{ Valor-p } & \multicolumn{3}{|c|}{ Intervenção } & \multirow{3}{*}{ Valor-p } \\
\hline \multirow{2}{*}{ Classificação } & $\mathrm{C} 1$ & $\mathrm{C} 2$ & C3 & & $\mathrm{C} 1$ & $\mathrm{C} 2$ & $\mathrm{C} 3$ & \\
\hline & n (\%) & n (\%) & $\mathrm{n}(\%)$ & & n (\%) & n (\%) & $\mathrm{n}(\%)$ & \\
\hline $\begin{array}{c}\text { O Não } \\
\text { aderente }\end{array}$ & $2(4,7)$ & $2(4,7)$ & $1(2,3)$ & & $4(9,3)$ & $4(9,3)$ & $2(4,7)$ & \\
\hline $\begin{array}{l}1 \text { Baixa } \\
\text { adesão }\end{array}$ & $15(34,9)$ & $14(32,6)$ & $20(46,5)$ & & $16(37,2)$ & $14(32,6)$ & $15(34,9)$ & \\
\hline $\begin{array}{c}2 \\
\text { Moderada } \\
\text { adesão }\end{array}$ & $9(20,9)$ & $10(23,3)$ & $12(27,9)$ & 0,26 & $7(16,3)$ & $8(18,6)$ & $5(11,6)$ & 0,35 \\
\hline $\begin{array}{c}3 \text { Máxima } \\
\text { adesão }\end{array}$ & $17(39,5)$ & $17(39,5)$ & $10(23,3)$ & & $16(37,2)$ & $17(39,5)$ & $21(48,8)$ & \\
\hline
\end{tabular}

Teste de Friedman. $\mathrm{C}$ = consulta

Fonte: Dados de Pesquisa.

Tabela 5. Comparação entre nível de atividade física e classificação de risco. Brasília, 2019.

\begin{tabular}{|c|c|c|c|c|c|c|c|c|c|c|c|c|}
\hline \multirow{3}{*}{$\begin{array}{c}\text { Classificação } \\
\text { de risco }\end{array}$} & \multicolumn{4}{|c|}{ Grupo } & \multicolumn{6}{|c|}{ IPAQ - Nível de atividade física } & & \\
\hline & \multicolumn{2}{|c|}{ Muito ativo } & \multicolumn{2}{|c|}{ Ativo } & \multicolumn{2}{|c|}{$\begin{array}{c}\text { Irregularmente } \\
\text { ativo } \mathrm{A}\end{array}$} & \multicolumn{2}{|c|}{$\begin{array}{c}\text { Irregularmente } \\
\text { ativo B }\end{array}$} & \multicolumn{2}{|c|}{ Sedentário } & \multicolumn{2}{|c|}{ Valor-p } \\
\hline & $\mathrm{N}$ & $\%$ & $\mathrm{~N}$ & $\%$ & $\mathrm{~N}$ & $\%$ & $\mathrm{~N}$ & $\%$ & $\mathrm{~N}$ & $\%$ & & \\
\hline Alto risco & \multirow{4}{*}{ Controle } & 0 & 0 & 3 & 8,82 & 0 & 0 & 0 & 0 & 0 & 0 & \multirow{4}{*}{0,043} \\
\hline Baixo risco & & 10 & 83,33 & 10 & 29,41 & 8 & 40 & 15 & 53,57 & 14 & 45,16 & \\
\hline $\begin{array}{l}\text { Médio } \\
\text { risco }\end{array}$ & & 1 & 8,33 & 19 & 55,88 & 11 & 55 & 13 & 46,42 & 16 & 51,61 & \\
\hline Sem risco & & 1 & 8,33 & 2 & 5,88 & 1 & 5 & 0 & 0 & 1 & 3,22 & \\
\hline Alto risco & \multirow{4}{*}{ Intervenção } & 0 & 0 & 0 & 0 & 0 & 0 & 0 & 0 & 0 & 0 & \multirow{4}{*}{0,026} \\
\hline Baixo risco & & 16 & 66,66 & 17 & 53,12 & 10 & 43,47 & 14 & 35,89 & 3 & 27,27 & \\
\hline Médio & & 4 & 16,66 & 12 & 37,5 & 9 & 39,13 & 23 & 58,97 & 8 & 72,72 & \\
\hline Sem risco & & 4 & 16,66 & 3 & 9,37 & 4 & 17,39 & 2 & 5,12 & 0 & 0 & \\
\hline
\end{tabular}

Teste exato de Fisher.

Fonte: Dados de Pesquisa.

Dessa forma, ressalta-se o papel da comunicação como um diferencial entre o paciente e os membros da equipe de saúde para que o gerenciamento da doença se estabeleça, assim como a adesão ao tratamento à medida que se proporciona a autopercepção da necessidade de tratamento pelo próprio paciente ${ }^{23}$, portanto, as consultas de enfermagem podem criar um ciclo de feedback e aumentar o envolvimento do paciente no processo saúde-doença ${ }^{22,24}$.

A maioria dos fatores de risco contribuintes para doença cardiovascular identificado na atual investigação é modificável. Esses fatores de risco devem ser conhecidos, monitorados e documentados para que as intervenções possam ser planejadas e implementadas pela equipe de saúde por meio de um trabalho colaborativo ${ }^{25}$. Dado que o risco cardiovascular, na atual investigação, interferiu inclusive na prática de atividade física, tanto no grupo intervenção $(p=0,0261)$ como no controle $(p=0,0438)$. Isso reforça a importância da prevenção primária de doenças cardiovasculares, mas doenças como a depressão, identificadas de forma proporcional entre os pacientes do grupo intervenção e controle podem dificultar o gerenciamento do cuidado ${ }^{26}$. Nessa vertente, o enfermeiro atuante em um modelo avançado de cuidado pode promover, inclusive o cuidado direcionado e contribuir para redução de taxas de depressão, como apresentado em uma revisão sistemática que aborda a atuação de enfermeiros no cuidado a longo prazo ${ }^{27}$, considerando que o exercício avançado de cuidado tem sido expandido ao sistema público de saúde ${ }^{28}$, 
inclusive no Brasil, como uma possibilidade de responder aos problemas decorrentes das necessidades de saúde.

O gerenciamento do cuidado inclui abordagens específicas, de acordo com a causa subjacente, para prevenir e controlar complicações ${ }^{29}$, a exemplo dos casos de risco, lesão e falência renal identificados dentre os pacientes, o que ressalta a importância de se reconhecer a LRA como uma patologia binária, ou seja, que pode evoluir com recuperação da função renal ou necessidade de diálise. Nesse contexto, profissionais de saúde, como os enfermeiros, podem desempenhar papel fundamental para o autogerenciamento do paciente, especialmente, daqueles com doenças crônicas por meio de um modelo de cuidado eficiente que possibilite a implementação de intervenções integradas no âmbito da atenção primária à saúde ${ }^{11}$. Esse tipo de modelo de cuidado contribuiu para o aumento do número de pacientes sem risco cardiovascular do grupo intervenção $(0,00 \%$ para $25,58 \%)$, já no grupo controle o número de pacientes sem risco reduziu de $6,98 \%$ para $2,33 \%$, o que evidencia maior sucesso no gerenciamento do grupo com monitoramento avançado (intervenção).

Nesse contexto, tem-se discutido a importância da atuação do enfermeiro de prática avançada, que integra em sua assistência pesquisa, educação, prática assistencial e gestão. Assim, esse enfermeiro deve ter autonomia profissional e competência para tomar decisões clínicas, realizar avaliações, diagnóstico e prescrições, sendo responsável pela gestão de casos, avaliação e implementação de programas e planos de cuidado, além de ser referência como primeiro ponto de contato dos usuários com os serviços de saúde ${ }^{28}$, condições essenciais na atenção primária.

O gerenciamento na atenção primária por meio de equipes de profissionais tem se mostrado um modelo promissor de cuidado que visa identificar pacientes de risco ${ }^{8}$, além de auxiliar no gerenciamento de medicamentos, educação sobre medicamentos e treinamento para melhorar a adesão medicamentosa ${ }^{30}$. Sabese que a adesão a medicamentos é um importante aspecto para eficácia do tratamento, custos de saúde e segurança do paciente ${ }^{8,31}$, também traduz a noção de concordância, ou seja, um processo de tomada de decisão compartilhada entre pacientes e profissionais de saúde. Muitos dos adultos mais velhos sofrem de várias doenças e, portanto, são tratados com diferentes medicamentos, o que determina maior risco de baixa adesão e exacerbação das doenças crônicas ${ }^{32,33}$. Nota-se que 0 número de não aderentes reduziu, proporcionalmente, nos dois grupos, controle e intervenção. Mas, a máxima adesão aumentou, principalmente, no grupo intervenção ( $37,2 \%$ para $48,8 \%$ ), ainda que sem significância estatística. A não adesão e a impossibilidade de comprar medicamentos contribuem para condições crônicas não controladas, intensificações desnecessárias da terapia e maior utilização dos serviços de saúde ${ }^{34,35}$.

Muitas orientações fornecidas em consultas são esquecidas por $40 \%$ a $80 \%$ dos pacientes ${ }^{36}$. Fatores como idade avançada ${ }^{37}$ e escolarização diminuída ${ }^{38,39}$ podem favorecer o processo de esquecimento, essas características foram encontradas entre os pacientes deste estudo. Nesse contexto, serviços de atenção primária, como na atual investigação, muitas vezes, com poucos recursos, devem concentrar esforços no desenvolvimento de modelos de monitoramento e estratificação de risco que incorpore dados da atenção primária e secundária, além de dados clínicos em tempo real, coletados durante a avaliação do paciente para melhor direcionamento e individualização do cuidado ${ }^{40}$.

Nesse sentido, a contribuição do acompanhamento de enfermagem com um trabalho informativo e de sensibilização no âmbito da saúde pública ${ }^{22}$, de forma intensificada, mostrou-se relevante, contribuindo para o autocuidado de usuários hipertensos e diabéticos, evidenciando a contribuição e importância destes profissionais no contexto da atenção primária à saúde ${ }^{41}$.

\section{CONCLUSÕES E IMPLICAÇÕES PARA A PRÁTICA}

A monitorização avançada possibilitou uma melhor identificação de pacientes de risco e orientações aos pacientes hipertensos e diabéticos com risco cardiovascular e renal, o que favoreceu o monitoramento avançado e contribuiu ao autogerenciamento da prática de exercícios e adesão medicamentosa a partir de consultas de enfermagem. Verificou-se também elevada taxa de fatores de risco modificáveis para doença cardiovascular, sendo seu impacto negativo para a prática da atividade física.

As limitações deste estudo se concentram em um estudo unicêntrico do tipo quase experimental, o que configura a impossibilidade de uma amostragem randomizada e, portanto, pode favorecer a ocorrência do risco de viés de seleção. No entanto, para minimizar esse risco, estabeleceu-se um critério imparcial baseado no número do registro de cadastro de cada paciente, reduzindo a chance de manipulação no processo de seleção. $A$ análise dos efeitos dos medicamentos não foi possível pela quantidade de dados ausentes e a função renal foi avaliada somente pela creatinina sérica por impossibilidade de obter precisamente o volume urinário.

Por outro lado, evidencia-se que o monitoramento avançado realizado pelos enfermeiros contribuiu ao autocuidado dos usuários pela possibilidade de ampliação da compreensão da importância desse modelo de cuidado guiado por consultas de enfermagem, estabelecimento de um ciclo de feedback e construção de vínculo de confiança no qual a comunicação quando foi associada ao controle intensificado de variáveis hemodinâmicas e laboratoriais mostrou-se ferramenta essencial e facilitadora da autopercepção da necessidade de cuidado, promoveu maior adesão ao tratamento, assim como o autogerenciamento do cuidado.

\section{FINANCIAMENTO}

Fundação de Apoio a Pesquisa do Distrito Federa (FAPDF) na modalidade seleção pública de propostas de pesquisa científica, tecnologia e inovação demanda espontânea, Brasília, Distrito Federal - Brasil, Edital 04/2017 FAP-DF, projeto intitulado: Implementação de um estudo para triagem da doença renal crônica para melhora dos cuidados de diabéticos e hipertensos na 
atenção primária à saúde. Processo: 16890.78.33839.26042017, concedido a Marcia Cristina da Silva Magro.

\section{CONTRIBUIÇÕES DOS AUTORES}

Desenho do estudo. Layse Farias Nava. Wellington Luiz de Lima. Marcia Cristina da Silva Magro.

Coleta de dados. Layse Farias Nava. Wellington Luiz de Lima. Marcia Cristina da Silva Magro. Tayse Tâmara da Paixão Duarte. Análise de dados. Layse Farias Nava. Wellington Luiz de Lima. Marcia Cristina da Silva Magro. Tayse Tâmara da Paixão Duarte. Interpretação dos resultados. Layse Farias Nava. Wellington Luiz de Lima. Marcia Cristina da Silva Magro. Tayse Tâmara da Paixão Duarte.

Redação e revisão crítica do manuscrito. Layse Farias Nava. Tayse Tâmara da Paixão Duarte. Wellington Luiz de Lima. Marcia Cristina da Silva Magro.

Aprovação da versão final do artigo. Layse Farias Nava. Tayse Tâmara da Paixão Duarte. Wellington Luiz de Lima. Marcia Cristina da Silva Magro.

Responsabilidade por todos os aspectos do conteúdo e a integridade do artigo publicado. Layse Farias Nava. Tayse Tâmara da Paixão Duarte. Wellington Luiz de Lima. Marcia Cristina da Silva Magro.

\section{EDITOR ASSOCIADO}

Maria Catarina Salvador da Motta (1)

\section{EDITOR CIENTÍFICO}

\author{
Ivone Evangelista Cabral (B)
}

\section{REFERÊNCIAS}

1. American Diabetes Association. 1. Improving care and promoting health in populations: Standards of medical care in diabetes - 2019 Diabetes Care. 2019 jan 17;42(Suppl 1):S7-12. http://dx.doi.org/10.2337/ dc19-S001. PMid:30559227.

2. ArafatY, Mohamed Ibrahim MI, Awaisu A, Colagiuri S, OwusuY, Morisky $\mathrm{DE}$ et al. Using the transtheoretical model's stages of change to predict medication adherence in patients with type 2 diabetes mellitus in a primary health care setting. Daru. 2019 jun 7;27(1):91-9. http://dx.doi. org/10.1007/s40199-019-00246-7. PMid:30729403.

3. Petersen M. Economic costs of diabetes in the U.S. in 2002. Diabetes Care. 2003;26(3):917-32. http://dx.doi.org/10.2337/diacare.26.3.917. PMid:12610059.

4. American Diabetes Association. Economic costs of diabetes in the U.S. in 2007. Diabetes Care. 2008 mar 1;31(3):596-615. http://dx.doi org/10.2337/dc08-9017. PMid:18308683.

5. Van Erp RMA, Van Doorn AL, Van Den Brink GT, Peters JWB, Laurant $\mathrm{MGH}$, Van Vught AJ. Physician Assistants and nurse practitioners in primary care plus: a systematic review. Int J Integr Care. $2021 \mathrm{fev}$ 12;21(1):6. http://dx.doi.org/10.5334/ijic.5485. PMid:33613138.

6. Lovink MH, Persoon A, Koopmans R, Van Vught A, Schoonhoven L, Laurant MGH. Effects of substituting nurse practitioners, physician assistants or nurses for physicians concerning healthcare for the ageing population: a systematic literature review. J Adv Nurs. 2017;73(9):2084102. http://dx.doi.org/10.1111/jan.13299. PMid:28299815.
7. Al Sadig AI Mahdi T. Overview of the course of undergraduate medical education in the Sudan. Sudan Journal of Medical Sciences. 2019 fev;14(4):188-201. http://dx.doi.org/10.18502/sjms.v14i4.5899.

8. Gonçalves JR, Gurgel CP. Cuidados e prevenções ao diabetes no Brasil. Revista JRG de Estudos Acadêmicos. 2019 jun;2(4):1-16. http://dx.doi. org/10.5281/zenodo.4321960.

9. Cross-Denny B, Robinson MA. Using the social determinants of health as a framework to examine and address predictors of depression in later life. Aging InteR. 2017;42(4):393-412. http://dx.doi.org/10.1007/ s12126-017-9278-6.

10. Dalmolin BB, Backes DS, Zamberlan C, Schaurich D, Colomé JS Gehlen MH. Significados do conceito de saúde na perspectiva de docentes da área da saúde. Esc Anna Nery. 2011 jun;15(2):389-94. http://dx.doi.org/10.1590/S1414-81452011000200023.

11. Dineen-Griffin S, Garcia-Cardenas V, Williams K, Benrimoj SI. Helping patients help themselves: a systematic review of self-management support strategies in primary health care practice. PLoS One. 2019 Aug 1;14(8):e0220116. http://dx.doi.org/10.1371/journal.pone.0220116.

12. Morisky DE, Green LW, Levine DM. Concurrent and predictive validity of a self-reported measure of medication adherence. Med Care. 1986;24(1):67-74. http://dx.doi.org/10.1097/00005650-19860100000007. PMid:3945130.

13. Matsudo S, Araújo T, Matsudo V, Andrade D, Andrade E, Oliveira LC, et al. Questionário internacional de atividade física (IPAQ): estudo de validade e reprodutibilidade no Brasil. Rev Bras Ativ Fís Saúde. 2012;6(2):5-18. http://dx.doi.org/10.12820/rbafs.v.6n2p5-18.

14. Hallal PC, Victora CG. Reliability and validity of the International Physical Activity Questionnaire (IPAQ). Med Sci Sports Exerc. 2004 mar;36(3):556. http://dx.doi.org/10.1249/01.MSS.0000117161.66394.07. PMid:15076800.

15. Barros ALBL. Anamnese e exame físico: avaliação diagnóstica de enfermagem no adulto. Porto Alegre: ARTMED; 2016.

16. Governo do Distrito Federal (BR). Secretaria de Estado e Saúde. Subsecretaria de Atenção Integral à Saúde. Comissão Permanente de Protocolos de Atenção à Saúde. Protocolo de Atenção à Saúde. Manejo da Hipertensão Arterial Sistêmica e Diabetes. Mellitus na Atenção Primária à Saúde [Internet]. Brasília (DF): Secretaria de Estado e Saúde; 2018 Disponivel em: http://www.saude.df.gov.br/wp-conteudo/uploads/2018/04/ hipertencao-e-diabetes-Manejo_da_HAS_e_DM_na_APS.pdf

17. Jane M, Hagger M, Foster J, Ho S, Kane R, Pal S. Effects of a weight management program delivered by social media on weight and metabolic syndrome risk factors in overweight and obese adults: a randomised controlled trial. PLoS One. 2017;12(6):e0178326. http:// dx.doi.org/10.1371/journal.pone.0178326.

18. D'Agostino Sr RB, Vasan RS, Pencina MJ, Wolf PA, Cobain M, Massaro $\mathrm{JM}$ et al. General cardiovascular risk profile for use in primary care: the Framingham heart study. Circulation. 2008 fev 12;117(6):743-53. http:// dx.doi.org/10.1161/CIRCULATIONAHA.107.699579. PMid:18212285.

19. Kellum JA, Lameire N, Aspelin P, Barsoum RS, Burdmann EA, Goldstein SL et al. Kidney Disease: Improving Global Outcomes (KDIGO). Working Group. Definition and classification of acute kidney injury. Kidney Int Suppl. [Internet]. 2012 [citado 2021 jun 15];2:19-36. Disponível em: https://kdigo.org/wp-content/uploads/2016/10/KDIGO-2012-AKIGuideline-English.pdf

20. Chawla LS, Bellomo R, Bihorac A, Goldstein SL, Siew ED, Bagshaw $S M$ et al. Acute kidney disease and renal recovery: consensus report of the Acute Disease Quality Initiative (ADQI) 16 Workgroup. Nat Rev Nephrol.2017;13(4):241-57. http://dx.doi.org/10.1038/nrneph.2017.2. PMid:28239173.

21. Norful A, Martsolf G, De Jacq K, Poghosyan L. Utilization of registered nurses in primary care teams: a systematic review. Int J Nurs Stud 2017;74:15-23. http://dx.doi.org/10.1016/j.ijnurstu.2017.05.013. PMid:28595110.

22. Cunha CMSLM, Henriques MAP, Costa AJS. A enfermagem de saúde pública e as políticas públicas de saúde: um estudo de caso. Esc Anna Nery. 2021;25(5):e20210204. http://dx.doi.org/10.1590/2177-9465ean-2021-0204 
23. Ledford CJW, Seehusen DA, Crawford PF. Toward a model of shared meaningful diagnosis. Patient Educ Couns. 2021 jan;104(1):143-8. http://dx.doi.org/10.1016/j.pec.2020.07.016. PMid:32771242.

24. Hong YR, Cardel M, Suk R, Vaughn IA, Deshmukh AA, Fisher CL et al. Teach-back experience and hospitalization risk among patients with ambulatory care sensitive conditions: a matched cohort study. J Gen Intern Med. 2019 out;34(10):2176-84. http://dx.doi.org/10.1007/s11606019-05135-y. PMid:31385206.

25. Karmali KN, Persell SD, Perel P, Lloyd-Jones DM, Berendsen MA, Huffman MD. Risk scoring for the primary prevention of cardiovascular disease. Cochrane Database Syst Rev. 2017;3(3):CD006887. http:// dx.doi.org/10.1002/14651858.CD006887.pub4. PMid:28290160.

26. O'Neill B, Kalia S, Aliarzadeh B, Sullivan F, Moineddin R, Kelly M et al. Cardiovascular risk factor documentation and management in primary care electronic medical records among people with schizophrenia in Ontario, Canada: Retrospective cohort study. BMJ Open. 2020 out 16;10(10):e038013. http://dx.doi.org/10.1136/bmjopen-2020-038013. PMid:33067284.

27. Donald F, Martin-Misener R, Carter N, Donald EE, Kaasalainen S, WicksonGriffiths A et al. A systematic review of the effectiveness of advanced practice nurses in long-term care. J Adv Nurs. 2013;69(10):2148-61. http://dx.doi.org/10.1111/jan.12140. PMid:23527481.

28. Miranda MV No, Rewa T, Leonello VM, Oliveira MAC. Advanced practice nursing: a possibility for Primary Health Care? Rev Bras Enferm. 2018;71(Suppl 1):716-21. http://dx.doi.org/10.1590/0034-7167-20170672. PMid:29562033.

29. Levey AS, James MT. Acute kidney. Ann Intern Med. 2017 nov 7;167(9):ITC6579. http://dx.doi.org/10.7326/AITC201711070. PMid:29114754.

30. Chang ET, Raja PV, Stockdale SE, Katz ML, Zulman DM, Eng JA et al. What are the key elements for implementing intensive primary care? A multisite Veterans Health Administration case study. Healthc (Amst). 2018 dez 1;6(4):231-7. http://dx.doi.org/10.1016/j.hjdsi.2017.10.001. PMid:29102480.

31. Osterberg L, Blaschke T. Adherence to medication. N Engl J Med. 2005 ago 4;353(5):487-97. http://dx.doi.org/10.1056/NEJMra050100. PMid: 16079372 .

32. Mansur N, Weiss A, Hoffman A, Gruenewald T, Beloosesky Y. Continuity and adherence to long-term drug treatment by geriatric patients after hospital discharge: a prospective cohort study. Drugs Aging 2008;25(10):861-70. http://dx.doi.org/10.2165/00002512-20082510000005. PMid: 18808210

33. Fallis BA, Dhalla IA, Klemensberg J, Bell CM. Primary medication nonadherence after discharge from a general internal medicine service. PLoS One. 2013 maio 2;8(5):e61735. http://dx.doi.org/10.1371/journal. pone.0061735. PMid:23658698.

34. Remondi FA, Cabrera MAS, de Souza RKT. Não adesão ao tratamento medicamentoso contínuo: Prevalência e determinantes em adultos de 40 anos e mais. Cad Saude Publica. 2014 jan;30(1):126-36. http:// dx.doi.org/10.1590/0102-311X00092613. PMid:24627020.

35. Tavares NUL, Bertoldi AD, Mengue SS, Arrais PSD, Luiza VL, Oliveira $M A$ et al. Factors associated with low adherence to medicine treatment for chronic diseases in brazil. Rev Saude Publica. 2016 dez 12;50(Supp 2):10s. http://dx.doi.org/10.1590/s1518-8787.2016050006150. PMid:27982378.

36. Sherlock A, Brownie S. Patients' recollection and understanding of informed consent: A literature review. ANZ J Surg. 2014 abr;84(4):20710. http://dx.doi.org/10.1111/ans.12555. PMid:24812707.

37. Jansen J, Butow PN, Van Weert JCM, Van Dulmen S, Devine RJ, Heeren $\mathrm{TJ}$ et al. Does age really matter? Recall of information presented to newly referred patients with cancer. J Clin Oncol. 2008 nov 20;26(33):5450-7. http://dx.doi.org/10.1200/JCO.2007.15.2322. PMid:18936478.

38. Selic P, Svab I, Repolusk M, Gucek NK. What factors affect patients' recall of general practitioners' advice? BMC Fam Pract. 2011 dez 28;12(1):141. http://dx.doi.org/10.1186/1471-2296-12-141. PMid:22204743.

39. McCarthy DM, Waite KR, Curtis LM, Engel KG, Baker DW, Wolf MS. What did the doctor say? Health literacy and recall of medical instructions. Med Care.2012 abr;50(4):277-82. http://dx.doi.org/10.1097/ MLR.0b013e318241e8e1. PMid:22411440.

40. Emmett L, Tollitt J, McCorkindale S, Sinha S, Poulikakos D. The evidence of acute kidney injury in the community and for primary care interventions. Nephron. 2017 jul 1;136(3):202-10. http://dx.doi.org/10.1159/000460266. PMid:28343224.

41. Cunha CMSLM, Henriques MAP, Costa AJS. Public health nursing: regulation and public health policies. Rev Bras Enferm. 2020;73(6):e20190550. http://dx.doi.org/10.1590/0034-7167-2019-0550. PMid:32901741. 